\title{
Enriquecimento ambiental e bem-estar na produção animal
}

\author{
Environmental enrichment and welfare in animal production
}

\section{Gisele Dela Ricci*, Cristiane Gonçalves Titto e Rafael Teixeira de Sousa}

Recebido em 28/01/2016 / Aceito em 19/04/2017

\section{RESUMO}

$\mathrm{O}$ atendimento do bem-estar animal está entre as principais exigências dos consumidores, que estão cada vez mais atentos a qualidade de vida dentro do ambiente criatório, o que exige o uso de condutas éticas nos sistemas destinados à produção animal. Dentro desta perspectiva este estudo teve como objetivo descrever a importância do enriquecimento ambiental na promoção da melhoria do bem-estar em animais de produção. O enriquecimento ambiental tem como principal característica reduzir estímulos que gerem respostas estressoras e permitir a apresentação de comportamentos naturais, evitando o aparecimento de comportamentos estereotipados em animais confinados. O comportamento natural foi definido como aquele em que o animal tende a realizar livre, por trazer prazer ou promover bom funcionamento fisiológico. Enquanto que o bem-estar animal esta relacionado às condições do alojamento como a bioclimatologia e a biofísica das instalações, podendo ser mensurado a partir de avaliações fisiológicas e comportamentais. O estresse é tido como um conjunto de reações fisiológicas intensas e prolongadas que resultam em desequilíbrio da homeostase, com efeitos importantes e irreversíveis aos animais. Deste modo, conclui-se que o uso enriquecimento ambiental pode contribuir grandemente na redução dos fatores estressores, melhorando o bem-estar em sistemas de produção animal.

PALAVRAS-CHAVE: ruminantes, suínos, aves, estresse, comportamento animal.

\section{ABSTRACT}

Animal welfare is among the main demands of consumers, who are increasingly critical of the quality of life within the environment, which requires the use of static conducts in systems for animal production. From this perspective, the objective of this study was to describe the importance of environmental enrichment in promoting improved welfare in animal production. Environmental enrichment has as main characteristic to reduce stimuli that generate stressful responses and allow the presentation of natural behavior, avoiding the appearance of stereotyped behaviors in confined animals. Natural behavior was defined as the one in which the animal tends to perform freely, by bringing pleasure or promoting good physiological functioning. While animal welfare is related to housing conditions such as bioclimatology and plant biophysics, it can be measured from physiological and behavioral assessments. Stress is seen as a set of intense and prolonged physiological reactions that result in imbalance of homeostasis, with important and irreversible effects on the animals. Thus, it is concluded that the use of environmental enrichment can greatly contribute to the reduction of stressors, improving animal welfare in production systems.

KEYWORDS: ruminants, swine, poultry, stress, animal behavior.

\section{INTRODUÇÃO}

O confinamento surgiu como alternativa eficiente para monitorar os animais de produção, por reduzir as perdas energéticas, de espaço e mão de obra, aumentando a produtividade assim como os problemas relacionados ao comportamento e qualidade de vida dos animais (MACHADO FILHO \& HÖTZEL 2000). Nesses sistemas de criação o comportamento possui relação importante com o ambiente, tornando-se um indicador das adaptações fisiológicas e biológicas, representando a parte de interação com o habitat (SNOWDON 1999). Sendo este controlado a partir de mecanismos neurobiológicos e hormonais, ocorrendo

Universidade de São Paulo, Pirassununga, SP, Brasil.

*Autor para correspondência < giseledelaricci@usp.br> 
tanto individualmente quanto em grupos, o que o torna uma ferramenta importante no que tange a melhorias dos fatores promotores do bem-estar (MENCH 1992).

Concomitantemente aos fatores anteriormente citados, o bem-estar animal, está associado a questões ligadas à utilização adequada dos recursos ambientais e a segurança alimentar tem recebido atenção da sociedade, estabelecendo novos desafios para as atividades agropecuárias (FRASER \& BROOM 1990). Entre as principais exigências dos consumidores está a qualidade de vida dentro do confinamento, que exige a criação ética dos animais destinados à produção (POLETTO 2009).

Dentro desse contexto o enriquecimento ambiental surge como uma possibilidade de melhoria do bem-estar animal nos sistemas de confinamento (DALLA COSTA et al. 2005), proporcionando aos animais um ambiente mais adequado para criação, visando maior qualidade de vida por meio da expressão comportamental típicos dos animais, onde quanto mais opções de expressão de comportamentos naturais, maiores serão as chances de sucesso ao lidar com fatores estressantes em seu ambiente (PINHEIRO 2009).

Sabendo-se da importância do enriquecimento ambiental para o bem-estar animal, esta revisão tem como objetivo descrever e caracterizar o uso dessas ferramentas na melhoria do sistema criatório em animais de produção.

\section{DESENVOLVIMENTO}

Os métodos de criação sofreram mudanças significativas relacionadas principalmente ao número excessivo de animais alocados em baias de tamanho reduzido, cujo o advento ocorreu principalmente no período pós-segunda guerra mundial (GONYOU 1994, BROOM \& FRASER 2010). Entretanto, recentemente aumentou-se a preocupação como o histórico de produção do produto final, do nascimento da matéria prima até a sua comercialização nas prateleiras dos supermercados, e esse estímulo se deu em decorrência do interesse do público pela forma como os animais são tratados e criados (FRASER 2000). Nesse aspecto o aperfeiçoamento de técnicas e pesquisas relacionadas ao bem-estar para animais de produção tendem a gerar benefícios que proporcionam aumento da qualidade de vida dentro das criações (PEDERSEN et al. 2002, HOLM et al. 2002).

No Brasil, pesquisas tem se desenvolvido para avaliar o comportamento típico das espécies em condições naturais buscando aperfeiçoar as práticas dentro dos confinamentos (NOGUEIRA et al. 2010) uma vez que mantê-lo torna-se essencial para a qualidade de vida dos animais (CARLSTEAD 1996).

O termo bem-estar foi descrito como o estado de um indivíduo e suas tentativas de se adaptar ao ambiente (BROOM 1986). O Comitê de Brambell também definiu bem-estar animal, na Inglaterra, por John Webster e adotado pelo Farm Animal Welfare Council (FAWC), fundamentando-se em cinco liberdades inerentes aos animais e entre elas estão à liberdade fisiológica, ambiental, sanitária, comportamental e psicológica (SILVA \& MIRANDA 2009, GRANDIN \& JOHNSON 2010). FRASER et al. (1997) propondo que o bem-estar contempla sentimentos, o funcionamento biológico e adaptações naturais. Deste modo, comportamentos naturais como alimentares, de reprodução, locomoção, lúdicos, descanso e típicos, como fuçar e construir ninhos em suínos, por exemplo, geram sentimentos positivos e consequentemente boa saúde (fisiológica e psicológica) (FRASER et al. 1997). A carência de comportamentos naturais induz os animais a frustração e aparecimento de comportamentos anômalos, como a agressividade e estereotipias (MASON et al. 2007).

O bem-estar pode ser mensurado a partir de avaliações fisiológicas e comportamentais. Medidas de frequência cardíaca, atividades das adrenais e respostas imunológicas (BROOM \& MOLENTO 2004) estão associadas a respostas de curto prazo e são mensuráveis de maneira objetiva (BROOM \& JOHNSON 1993). Comportamentos anormais como as estereotipias, a automutilação, o canibalismo, a agressividade excessiva e a apatia são, em conjunto, características que demonstram o seu bem-estar pobre entre os animais (ZANELLA 1995, BROOM \& MOLENTO 2004).

Para que um método de diagnóstico de bemestar animal seja eficiente, questões como praticidade, repetibilidade, confiabilidade e fatores externos devem ser considerados (BUTTERWORTH et al. 2011). O bem-estar pode ser definido quando o desempenho dos animais estiver de acordo com a sua qualidade de vida, ou seja, quando estiverem isentos de dores e vivendo em ambientes adequados (FRASER et al. 1997). Para isso, o mesmo precisa ser mensurado de forma objetiva buscando detectar as principais características que indicaram quais as mais efetivas medidas éticas perante a situações diversas (BROOM 
\& MOLENTO 2004), uma vez que este parâmetro pode variar de muito alto a muito baixo (BROOM \& FRASER 2010).

As políticas de desenvolvimento agrícola em vigor precisam considerar a necessidade de pequenos produtores, que praticam a atividade visando exportações, criando oportunidades reais, para o aumento dessas atividades, considerando especialmente o bem-estar, a sociedade e o ambiente (MAPA 2011, SILVA 2012). As legislações acerca do bem-estar animal influenciam diretamente as exportações de carne de frango e suína no Brasil, visto que os países importadores, em grande parte, são membros da União Europeia e estão exigindo qualidade ética na produção de animais destinados ao consumo (SILVA 2012). O sofrimento animal, desde a fazenda da criação até o momento do abate no frigorífico, tem sido foco de atenção buscando novos conceitos para adequar os sistemas de produção animal (WSPA 2007). A Normativa $n^{\circ} 65$ de 2008 orienta as boas práticas de bem-estar direcionado aos animais de produção, destacando especialmente o cuidado com práticas de manejo, que reduzam o estresse e consecutivos sofrimentos desnecessários (MAPA 2008).

Quaisquer restrições geradas pelo estresse da mudança de ambiente ou pelo confinamento causam alterações importantes no comportamento, na psicologia e fisiologia animal (SCHEPOP 2008). O comportamento natural foi definido como aquele em que o animal tende a realizar livre, por trazer prazer ou promover bom funcionamento fisiológico (MACHADO FILHO \& HÖTZEL 2000). A análise do comportamento animal é fundamental na adaptação biológica e representa a interação do indivíduo com o ambiente (SNOWDON 1999). A análise das alterações comportamentais é tida como um método prático e simples de avaliação do bem-estar (POLETTO 2010) em relação ao seu ambiente (BROOM 1991).

O conhecimento do comportamento natural pode ajudar no diagnóstico e aperfeiçoar o bemestar (FRASER 1993) através de alterações de postura, temperamento, sanidade indicando dores agudas e crônicas permitindo ainda a quantificação delas (MELLOR \& STAFFORD 2004). E dentre essas alterações o diagnóstico do comportamento estereotipado, torna-se um importante indicador do bem-estar, já que é considerado uma atividade anormal não apresentado por animais livres (BOSSO 2008). As estereotipias não têm um objetivo específico sendo repetido seguindo sempre um padrão e em uma sequência (XENOFONTE et al. 2006, BOSSO 2008, SCHEPOP 2008). São tentativas do animal em controlar seu ambiente, buscando ter maior controle sobre ele, a partir de comportamentos regulatórios específicos (SELLINGER \& HA 2005). No momento em que as estereotipias são exercidas pelos animais é possível através de medidas de manejo capazes de amenizá-la e ou eliminá-las. No entanto, se a frustração permanecer o comportamento será apresentado com maior frequência (BOSSO 2008). O comportamento estereotipado surge em animais com problemas permanentes relacionados ao ambiente, tornando-se uma tentativa de redução do dano causado ou demonstrar a alteração psicológica, indicando determinada alteração de saúde (BROOM \& KENNEDY 1993).

Tratando-se do desenvolvimento evolutivo dos equinos, espera-se que a ociosidade do confinamento fosse causa prioritária do aparecimento de estereotipias nestes animais. Atividades como roer madeira, aerofagia com e sem apoio e síndrome do urso são movimentos repetidos, sem função aparente, que podem ser observados prontamente (BROOM \& KENNEDY 1993). Equinos confinados apresentam com frequência significativa estereotipias com redução ou desaparecimento quando há inserção dos animais em áreas com maiores dimensões (BROOM \& KENNEDY 1993).

Em análise de leitões submetidos aos tratamentos de manutenção da leitegada na baia maternidade, a transferência de uma leitegada para a creche e a mistura de duas leitegadas de nove a 12 semanas alojadas na creche, foi observado que a mistura de lactentes com origens distintas afetou de forma mais significativa o bem-estar dos animais, notado principalmente pela inquietação e agressividade mais aparente. Notadamente, a mudança de ambiente colabora durante a fase de desmame para a apresentação elevada de comportamentos atípicos dos suínos. Manter os leitões nos mesmos lotes, desde a maternidade, apresenta maior concentração de comportamentos naturais (SOUZA 2007).

Para coelhos, o bem-estar depende, entre outros fatores, do espaço dentro das gaiolas uma vez que gaiolas pequenas ou uma lotação superior à adequada pode impedir a apresentação de comportamentos naturais, alterando além do comportamento, as funções sanitárias e produtivas (ARVEUX 1991). O comportamento lúdico destes animais apesar de 
fácil observação possui difícil definição (BEKOFF \& ALLEN 1998), sendo aceitos usualmente brinquedos objetos (madeiras, por exemplo), de locomoção (pular e arrastar) e sociais (entre indivíduos) (WALTERS 1987, BURGHART 1998).

Para ruminantes é imprescindível que os produtores e técnicos tenham amplo conhecimento sobre o comportamento natural das fases que estarão em contato, buscando compreender as diferentes interações entre animais e ambientes que quando negativas induzem a prejuízos econômicos importantes (BROOM \& JOHNSON 1993). Ovinos e caprinos são animais gregários e este pode ser o melhor entretenimento para estes animais. Brinquedos como bolas, correntes também podem ser utilizados, no entanto alguns animais podem não se interessar por este tipo de enriquecimento ambiental (VIEIRA 2014). Blocos nutricionais estão entre as opções para ruminantes, que além de fornecer dietas mais ricas, enriquecem o ambiente reduzindo o estresse dos animais confinados (FAPESP 2008).

É necessário que as instalações para criação animal sejam planejadas adequadamente com o aperfeiçoamento das práticas de manejo para minimizar o estresse acarretando o bem-estar dos animais (OLIVEIRA et al. 2001, BUENO et al. 2001), uma vez que animais em ambientes restritos tem sua qualidade de vida reduzida (SILVA et al. 2008). Tornando o ambiente adequado com a construção e manutenção das instalações, o ambiente permite a demonstração de comportamentos como o tédio e estereotipias que são indicadores específicos de bemestar pobre (BOSSO 2008).

$\mathrm{O}$ bem-estar intimamente relacionado às condições do alojamento como a bioclimatologia e a biofísica das instalações tanto para os animais quanto para os tratadores. Sendo que condições adequadas para os trabalhadores são aspectos importantes para a qualidade de vida dentro da produção animal (LIMA et al. 2004). E quando esses aspectos são respeitados ambas as partes são beneficiadas. Qualquer sistema de criação de aves pode ter suas instalações aperfeiçoadas, independentemente do tipo de tecnologia (ABREU \& ABREU 2011). Ovinos e caprinos, por exemplo, tem grande capacidade de se acostumar com os tratadores e quando existe a possibilidade de estabelecimento de uma interação positiva o estresse é reduzido de forma significativa (VIEIRA 2014). Em todas as espécies domésticas, a melhoria dos confinamentos, alimentação e a adequação dos métodos de manejo são, a priori, as ações que impedirão o desenvolvimento de atividades relativas a este estresse (BROOM \& KENNEDY 1993).

A agricultura orgânica, que surge como alternativa para o sistema convencional de produção, a partir de respeito à integridade, harmonia e individualidade dos animais (ALROE et al. 2001, LUND \& RÖCKLINSBERG 2001), tem dificuldades relacionadas ao bem-estar dos animais, principalmente relacionados a limitação no uso de drogas que controlem enfermidades e a não adequação de genótipos e seu ambiente de criação (CABARET et al. 2002, VAARST et al. 2001).

$\mathrm{Na}$ Europa, os produtores e a indústria estão continuamente efetuando alterações e adaptações nos sistemas de criação buscando o atendimento as legislações que atendem as exigências do mercado consumidor diante do bem-estar animal, enquanto na América do Norte, as alterações são recebidas a partir de mecanismos mercadológicos, que impõe suas diretivas diretamente aos produtores (FRASER 2006, TUYTTENS et al. 2008). Enquanto que no Brasil, a pressão por mudanças surge a partir de exigências dos mercados importadores, no entanto as regras internacionais limitam a comercialização de produtos de origem animal para os países com exigências maiores em relação a questões éticas da produção. Porém, observa-se que a população urbana brasileira tem demonstrado preocupação com a qualidade ética dos produtos disponíveis para consumo (MACHADO FILHO 2000).

\section{Enriquecimento ambiental}

O enriquecimento ambiental teve seus estudos ampliados na década de 1960 (VAN DE WEERD \& DAY 2009), voltados para animais de zoológicos e atualmente se estendeu para animais de laboratório e destinados a produção (ICKES et al. 2000, VAN de WEERD et al. 2003, CAMPOS et al. 2010). E o seu uso tem como objetivo reduzir estímulos que gerem respostas estressoras e permitam a apresentação de comportamentos naturais, evitando o aparecimento de comportamentos estereotipados em animais cativos (MENDONÇA-FURTADO 2006).

A redução de distúrbios comportamentais, do estresse, intervenções clínicas, mortalidade e o aumento das taxas reprodutivas são outros importantes benefícios do enriquecimento (CARLSTEAD \& SHEPHERDSON 2000). O enriquecimento são modificações no ambiente social e físico em que 
os animais estão inseridos que combinados com o habitat e comportamento típico, aumentam a qualidade e prevalência do bem-estar, a partir da saúde, comportamento e desempenho reprodutivo (BOERE 2001).

Entre as formas de inserção de enriquecimentos ambientais são citados objetos como pedaços de madeiras, cordas, pedras, escadas e pneus; ou alimentação diversa, a partir de alimentos diferentes aos do cotidiano como frutas ou legumes em diferentes formas como congelados, pendurados, cortados inteiros em horários alternados, como nos finais de tarde ou noite; e os enriquecimentos sensoriais, a partir de essências, com brinquedos para gustação, por exemplo, ervas (ALMEIDA et al. 2008, PINHEIRO 2009, CAMPOS et. al. 2010, MENDES et al. 2011) como a erva doce ou canela.

A disponibilização de enriquecimento ambiental diminui a incidência de caudofagia, consideradas interações agonísticas (GUY et al. 2002, RODARTE et al. 2004). A inserção de bolas ou brinquedos constituídos de borracha reduzem o tédio e aumento interações sociais lúdicas, por exemplo, de suínos (GUY et al. 2002). Segundo BEATTIE et al. (2000) suínos em ambientes enriquecidos direcionam um quarto do seu tempo em comportamentos no substrato do solo, enquanto animais em ambientes monótonos direcionam seu tempo a objetos fixos na baia e comportamentos sociais agressivos.

Para ABREU et al. (2006), a inserção de brinquedos para poedeiras pesadas em gaiolas enriquecidas há melhora do bem-estar a partir do comportamento mais calmo destes animais, porém não há influência na melhora da produção ou classificação de ovos bons em relação aos defeituosos. Para frangos de corte, a inserção de poleiros como enriquecimento, resultou em melhora da liberdade de comportamento no terço final da vida das aves, no entanto não houve efeitos significativos para os parâmetros zootécnicos avaliados (NORDI et al. 2006). Avaliando a eficiência do enriquecimento para coelhos, VERGA et al. (2004) indicaram diferenças no comportamento lúdico comparando animais mantidos em gaiolas com palha e outros em gaiolas convencionais.

Outro importante meio de enriquecer o ambiente buscando reduzir o estresse é a musicoterapia. A utilização de estímulos sensoriais, como também a aromaterapia, colaboram eficientemente para melhoria da qualidade de vida dos animais e dos seres humanos, embora até hoje tenha sido pouco estudada, já existem resultados promissores para redução de monotonia ambiental (ELLIS \& WELLS et al. 2010, MOREIRA et al. 2012).

A música pode permitir reabilitação física, mental e social de pessoas de forma individual e em grupos, uma vez que é uma mistura de diversas notas, amplitudes e tons, com sons contínuos e rítmicos, tornando-se um objeto relaxante sobre o indivíduo (MAIA et al. 2013). A música tem a capacidade de direcionar ao cérebro informações importantes para serem processadas, acarretando a ativação de regiões cerebrais responsáveis por estimular mudanças cognitivas e motoras (MOREIRA et al. 2012).

Relacionado à capacidade cognitiva dos animais, a música é capaz de influenciar no comportamento dos animais, a partir de reforços positivos e negativos (SARUBBI 2011). Avaliando a musica clássica ALBRIGHT \& ARAVE (1997) indicaram aumento da produção leiteira. JONES (2002) observando a influencia da utilização de rádio para aves, indicaram redução de comportamentos agonístico entre os animais.

Para a avaliação da eficiência do enriquecimento ambiental podem ser avaliadas alterações fisiológicas e comportamentais. As alterações fisiológicas podem ser medidas por meio de células de defesa do organismo, que são indicadores importantes para os animais, como por exemplo, para frangos de corte (GROSS \& SIEGEL 1983). As aves domésticas atuais são mantidas confinadas, no entanto estas mantêm características típicas dos ancestrais, como ciscar, por exemplo, sendo a observação comportamental importante para avaliação do bem-estar destes animais (DAWKINS 1999). Alterações comportamentais são indicações positivas da introdução de enriquecimentos (MAKI et al. 1989), uma vez que este procedimento deixa o ambiente mais complexo, tornando o confinamento menos tedioso (ALMEIDA 2006).

\section{CONCLUSÃO}

$\mathrm{O}$ enriquecimento ambiental associado ao comportamento animal apresenta-se de fundamental importância para a compreensão e melhoria do bemestar dos animais. Portanto, a análise das instalações e do comportamento é imprescindível para a manutenção da qualidade de vida e na prevenção de sofrimentos desnecessários dos animais destinados à produção. 


\section{REFERÊNCIAS}

ABREU VMN et al. 2006. Enriquecimento ambiental de gaiolas como estratégia prática para incrementar o bemestar e a produção de ovos de poedeiras pesadas. Concórdia: Embrapa. 5p. (Comunicado Técnico, 447).

ABREU VMN \& ABREU PG. 2011. Os desafios da ambiência sobre os sistemas de aves no Brasil. Revista Brasileira de Zootecnia 40: 1-14.

ALBRIGHT JL \& ARAVE CW. 1997. The behaviour of cattle. Cambridge: CAB International. 320p.

ALMEIDA AMR. 2006. Influência do enriquecimento ambiental no comportamento de primatas do gênero Ateles em diferentes condições de cativeiro no Departamento Zoológico de Curitiba, PR. Monografia (Bacharel em Ciências Biológicas). Curitiba: UFPR. 37p.

ALMEIDA AMR et al. 2008. Influência do enriquecimento ambiental no comportamento de primatas do gênero Ateles em cativeiro. Arquivos de Ciências Veterinárias e Zoologia da UNIPAR. 11: 97-102.

ALROE HF et al. 2001. Does organic farming face distinctive livestock welfare issues? A conceptual analysis. Journal of Agricultural and Environmental Ethics 14: 275299.

ARVEUX P. 1991. Densidad en jaulas de engorde. L'eleveur de Lapins 34: 66-67

BEATTIE VE et al. 2000. Influence of environmental enrichment on the behaviour, performance and meat quality of domestic pigs. Livestock Production Science 65: 71-79. BEKOFF M \& ALLEN C. 1998. Intentional communication and social play: how and why animals negotiate and agree to play. In: BEKOFF M \& BYERS JA. Animal play: evolutionary, comparative, and ecological perspectives. New York: Cambridge University Press. p. 97-114.

BOERE V. 2001. Behavior and environmental enrichment. In: FOWLER ME \& CUBAS ZS. 2001. Biology, Medicine and Surgery of South American Wild Animals. Iowa: Iowa State Press University. p. 263-267.

BOSSO PL. 2008. Enriquecimento ambiental. Fundação Parque Zoológico de São Paulo. Disponível em: $<$ http:// www.zoologico.sp.gov.br/peca7.htm>. Acesso em: 26 mar. 2015.

BUENO MG et al. 2001. Cortisol fecal como índice de resposta ao estresse em Panthera onca. In: V Congresso e X Encontro da Associação Brasileira de Veterinários de Animais Selvagens. Anais.... São Paulo: ABRAVAS. p.71. BURGHART GM. 1998. The evolutionary origins of play revised: lessons from turtles. In: BEKOFF M \& BYERS JA. Animal play: evolutionary, comparative, and ecological perspectives. Cambridge and New York: Cambridge University. pp.1-26.

BUTTERWORTH A et al. 2011. Practical strategies to assess (and improve) welfare. In: APPLEBY MC et al. Animal Welfare. 2. ed. Oxfordshire: CABI International. p. 200-214.
BROOM DM. 1986. Indicators of poor welfare. British Veterinary Journal 142: 524-526.

BROOM DM. 1991. Animal welfare: concepts and measurement. Journal of Animal Science 69: 4167-4175. BROOM DM \& JOHNSON KG. 1993. Assessing welfare: Short-term response. In: BROOM DM \& JOHNSON KG. Stress and Animal Welfare. London: Chapman \& Hall. p. $87-110$.

BROOM DM \& KENNEDY MJ. 1993. Stereotypies in Horses: their relevance to welfare and causation. Equine Veterinary Education 5: 151-154.

BROOM DM \& MOLENTO CFM. 2004. Bem-estar animal: conceito e questões relacionadas - Revisão. Archives of Veterinary Science 9: 1-11.

BROOM DM \& FRASER AF. 2010. Comportamento e bem-estar de animais domésticos. 4.ed. Barueri: Manole. 452p.

CABARET J et al. 2002. Managing helminths of ruminants in organic farming. Veterinary Research 33: 625-640.

CAMPOS JA et al. 2010. Enriquecimento ambiental para leitões na fase de creches advindos de desmame aos $21 \mathrm{e}$ 28 dias. Revista Brasileira de Ciências Agrárias 5: 272-278. CARLSTEAD K. 1996. Effects of captivity on the behavior of wild mammals. In: KLEIMAN DG et al. (Eds.). Wild Mammals in Captivity principles and techniques. Chicago: University of Chicago Press. p. 317-333.

CARLSTEAD K \& SHEPHERDSON D. 2000. Alleviating stress in zoo animals with environmental enrichment. In: MOBERG GP \& MENCH JA. The Biology of animal stress: basic principles and implications for animal welfare. [S.1.]: CAB International. p. 337-354.

DALLA COSTA OA et al. 2005. Aspectos econômicos e de bem-estar animal no manejo dos suínos da granja até o abate. In: 4 Seminário Internacional de Aves e Suínos. Anais... Florianópolis: UFSC. 25p.

DAWKINS MS. 1999. The role of behaviour in the assessment of poultry welfare. World's Poultry Science Journal 55: 295-303.

ELLIS SLH \& WELLS DL. 2010. The influence of olfactory stimulation on the behaviour of cats housed in a rescue shelter. Applied Animal Behaviour Science 123: 56-62.

FAPESP. 2008. Blocos Nutricionais para o rebanho. Disponível em: http://revistapesquisa.fapesp.br/. Acesso em: 04 jan. 2016.

FRASER AF. 1993. Assessing animal well-being: common sense, uncommon science. In: FOOD Animal Wellbeing. West Lafayette: USDA; Purdue University Press. p. 37-54. FRASER AF \& BROOM DM. 1990. Farm animal behaviour and welfare. 1.ed. Oxon: CABI. 437p.

FRASER D et al. 1997. A scientific conception of animal welfare that reflects ethical concerns. Animal Welfare 6: 187-205.

FRASER D. 2000. Animal ethics and animal welfare science: bridging the two cultures. Applied Animal 
Behaviour Science 65: 171-189.

FRASER D. 2006. Animal welfare assurance programs in food production: a framework for assessing the options. Animal Welfare 15: 93-104.

GONYOU HW. 1994. Why the study of animal behavior is associated with the animal welfare issue. Journal of Animal Science 72: 2171-2177.

GUY JH et al. 2002. Behaviour of two genotypes of growing-finishing pigs in three different housing systems. Applied Animal Behaviour Science 75: 193-206.

GRANDIN T \& JOHNSON C. 2010. O bem-estar dos animais - Proposta de uma vida melhor para todos os bichos. São Paulo: Rocco. 334p.

GROSS WB \& SIEGEL HS. 1983. Evaluation of the heterophil:lymphcyte ratio as a measure of stress in chickens. Avian Diseases 27: 972-979.

HOLM L et al. 2002. Calves' motivation for access to two different types of social contact measured by operant conditioning. Applied Animal Behaviour Science 79: 175194.

ICKES BR et al. 2000. Long-Term Environmental Enrichment Leads to Regional Increases in Neurotrophin Levels in Rat Brain. Experimental Neurology 164: 45-52.

JONES BR. 2002. Role of Comparative Psychology in the Development of Effective Environmental Enrichment Strategies to Improve Poultry Welfare. International Journal of Comparative Psychology 15: 77-106.

LIMA AMC et al. 2004. Ambiência e bem-estar. In: MENDES AA et al. (Orgs.). Produção de Frangos de Corte. 1.ed. Campinas: Facta. pp. 38-46.

LUND V \& RÖCKLINSBERG H. 2001. Outlining a conception of animal welfare for organic farming systems. Journal of Agricultural and Environmental Ethics 14: 391424.

MAIA APA et al. 2013. Enriquecimento ambiental como medida para o bem estar positivo de suínos - revisão. Revista Eletrônica em Gestão, Educação e Tecnologia Ambiental 14: 2862-2877.

MACHADO FILHO LCP. 2000. Pig welfare and meat quality: a Brazilian view. In International Virtual Conference on Pig Meat Quality. Anais... Concórdia: CNPSA/EMBRAPA. p. 98-118.

MACHADO FILHO LCP \& HÖTZEL MJ. 2000. Bem-estar em suínos. In: Seminário Internacional de Suinocultura. Anais... São Paulo: Gessuli. p. 88-105.

MAKI S et al. 1989. Food puzzle device simulation termite fishing for captive chimpanzee (Pan troglodytes). American Journal of Primatology Supplement 1: 71-78

MASON G et al. 2007. Why and how should we use environmental enrichment to tackle stereotypic behaviour? Applied Animal Behaviour Science 102: 163-188.

MENDES RC et al. 2011. Enriquecimento ambiental para leitões na fase de creche no IF Sudeste MG-Campus Barbacena. In: 2 Simpósio de Pesquisa e Inovação / 1 Seminário de Iniciação Científica. Anais.... Barbacena: IF
Sudeste MG. p. 22-24.

MENCH JA. 1992. Applied Ethology and poultry production. Poultry Science 71: 631-633.

MENDONÇA-FURTADO O. 2006. Uso de ferramentas como enriquecimento ambiental para macacos-prego (Cebus apella) cativos. Dissertação (Mestrado em Psicologia). São Paulo: USP. 92p.

MELLOR DJ \& STAFFORD KJ. 2004. Physiological and behavioural assessment of pain in ruminants: principles and caveats. ATLA Fourth World Congress 32: 267- 271. MAPA - Ministério da Agricultura, Pecuária e Abastecimento. 2008. Instrução Normativa $N^{\circ}$ 56, DE 6 de novembro de 2008. Disponível em: http://www.agricultura. gov.br/arq_editor/file/Desenvolvimento_Sustentavel/ Producao-Integrada-Pecuaria/IN\%2056\%20de\%202008. pdf. Acesso em: 22 jan. 2016.

MAPA - Ministério da Agricultura, Pecuária e Abastecimento. 2011. Agenda Estratégica 2010-2015: Aves e Suínos. Brasília. p. 41. Disponível em: < http:// www.agricultura.gov.br>. Acesso em: 02 maio de 2013.

MOREIRA SV et al. 2012. Neuromusicoterapia no Brasil: aspectos terapêuticos na reabilitação neurológica. Revista Brasileira de Musicoterapia 12: 18-26.

NOGUEIRA SSC et al. 2010. Social behavior of collared peccaries (Pecari tajacu) under three space allowances. Animal Welfare 19: 243-248.

NORDI WM et al. 2006. Impacto da oferta de poleiros sobre o bem-estar de frangos de corte. Archives of Veterinary Science 11: 19-25.

OLIVEIRA TG et al. 2001. Order Carnivora, Family Felidae (Cats). In: FOWLER ME (Ed.) Biology, Medicine, and Surgery of South American Wild Animals. Iowa State University Press: Ames. p. 291-316.

PEDERSEN LJ et al. 2002. Social isolation affects the motivation to work for food and straw in pigs as measured by operant conditioning techniques. Applied Animal Behaviour Science 77: 295-309.

PINHEIRO JV. 2009. A pesquisa com bem estar animal tendo como alicerce o enriquecimento ambiental através da utilização de objeto suspenso no comportamento de leitões desmamados e seu efeito como novidade. Dissertação (Mestrado em Ciências). São Paulo: USP. 65p.

POLETTO R. 2009. Série Especial: Bem-estar animal. Disponível em: http:/www.suino.com.br/Noticia/serieespecial-bem-estar-animal-por-rosangela-poletto-134530. Acesso em: 16 mai. 2017.

POLETTO R. 2010. Bem-estar animal. Suíno.com, Tangará, 5 abr. 2010. Série especial bem-estar animal por Rosangela Poletto. Disponível em: <http://tinyurl. com/4t6z4bk >. Online. Acesso em: 25 jul. 2010.

RODARTE LF et al. 2004. The effect of environmental manipulation on behavior, salivary cortisol and growth of piglets weaned at 14 days of age. Journal of Applied Animal Welfare Science 7: 171-179.

SARUBBI J. 2011. Bem estar animal não se restringe às 
instalações e equipamentos: o uso de novas tecnologias. In: 1 Fórum Integral de Suinocultura: Teoria e Prática do Bem Estar Animal na Produção de Suínos. Anais... Curitiba. p. 36-50.

SELLINGER RL \& HA JC. 2005. The effects of visitor density and intensity on the behavior of two captive jaguars (Panthera onca). Journal of Applied Animal Welfare Science 8: 233-244.

SILVA IJO et al. 2008. Influência do sistema de alojamento no comportamento e bem-estar de matrizes suínas em gestação. Revista Brasileira de Zootecnia 37: 1319-1329.

SILVA IJO \& MIRANDA KOS. 2009. Impactos do bemestar na produção de ovos. Thesis 6: 89-115.

SILVA RBTR. 2012. Itens normativos de bem-estar animal e a produção brasileira de frangos de corte. Tese (Doutorado em Engenharia Agrícola). Campinas: Unicamp. 84p.

SOUZA GPP. 2007. A influência de ambiente físico e social no bem-estar de leitões desmamados. Dissertação (Mestrado em Agroecossistemas). Florianópolis: UFSC. 85 p.

SCHEPOP JAV. 2008. Enriquecimento Ambiental. Disponível em <http://www.cativobemestaranimal.com.br/ enriquecimento.html>. Acesso em: 4 jan. 2016.

SNOWDON CT. 1999. O significado da pesquisa em comportamento animal. Estudos de Psicologia 4: 365-373. TUYTTENS FAM et al. 2008. Factors influencing farmers' responses to welfare legislation: A case study of gestation sow housing in Flanders (Belgium). Livestock Science 116: 289-299.

VAARST M et al. 2001. Health and welfare in Danish dairy cattle in the transition to organic production: Problems, priorities and perspectives. Journal of Agricultural and Environmental Ethics 14: 367-390.

VAN DE WEERD HA et al. 2003. A systematic approach towards developing environmental enrichment for pigs. Applied Animal Behaviour Science 84: 101-118.

VAN DE WEERD HA \& DAY JEL. 2009. A review of environmental enrichment for pigs housed in intensive housing systems. Applied Animal Behaviour Science 116: $1-20$.

VERGA M et al. 2004. Effect of housing, and environmental enrichment on performance and behaviour in fattening rabbits. In: 8 World Rabbit Congress. Proceedings... Puebla: World Rabbit Science Association/ Universidad Politecnica de Valencia. p.1283-1289. (CD-Rom).

VIEIRAFVR. 2014. Vale a pena ler de novo! Enriquecimento ambiental: uma eficiente ferramenta na produção de ovinos e caprinos. Disponível em: http://www.milkpoint.com.br/ radar-tecnico/ovinos-e-caprinos/vale-a-pena-ler-de-novoenriquecimento-ambiental-uma-eficiente-ferramenta-naproducao-de-ovinos-e-caprinos-61025n.aspx. Acesso: 22 jan. 2016.

WALTERS J. 1987. Transition to adulthood. In: SMUTS $\mathrm{B}$ et al. (Eds.). Primate societies. Chicago: University of Chicago. p.358-369.
WSPA - SOCIEDADE MUNDIAL DE PROTEÇÃO ANIMAL - WSPA. 2007. Entrevista: Bem-estar animal e desenvolvimento sustentável. Disponível em: . http://www. worldanimalprotection.org.br/ . Acesso em: 05 jan de 2016. XENOFONTE PF et al. 2006. Análise Comportamental de um casal de Panthera tigris tigris (Linnaeus, 1758) do Parque Dois Irmãos. In: 1 Encontro de Bioética e Bem Estar Animal do Agreste Meridional Pernambucano. Garanhuns. Pôsteres. Recife: UFRPE/UAG.

ZANELLA AJ. 1995. Indicadores fisiológicos e comportamentais do bem-estar animal. A Hora Veterinária 14: 47-52. 